\title{
On-chip Antenna: Practical Design and Characterization Considerations
}

\author{
A. Shamim, K. N. Salama \\ Department of Electrical Engineering \\ KAUST \\ Thuwal, Saudi Arabia
}

\author{
E. A. Soliman, S. Sedky \\ Dept. of Physics \\ AUC \\ Cairo, Egypt
}

\begin{abstract}
This paper highlights the challenges of an emergent field, namely, on-chip antenna design. Consistent with the RF System-on-Chip (SoC) concept, co-design strategy for circuits and on-chip antennas is described. A number of design and layout issues, arising from the highly integrated nature of this kind of systems, are discussed. The characterization difficulties related to on-chip antennas radiation properties are also highlighted. Finally, a novel on-wafer test fixture is proposed to measure the gain and radiation pattern of the on-chip antennas in the anechoic chamber.
\end{abstract}

Keywords- On-chip antenna; SoC; radiation pattern; IC

\section{INTRODUCTION}

The performance improvement in advanced CMOS technology has paved the way to RF component integration with analog and digital circuitry on a single chip. This progress of RF CMOS SoC has enabled highly compact handheld and portable devices. The attractiveness of these systems is that they can offer low cost, small physical size and low power consumption This concept has been widely accepted in applications like Wireless LAN and Bluetooth and is making inroads to cellular transceivers and GPS receivers[1]. It has also found its utility in many modern wireless sensor networks [2].

Miniaturization is a key factor in RF SoC design. In order to keep the transceiver size to a minimum, the antenna (which is the largest part of a typical transceiver module) has to be miniaturized. Traditionally, antennas are off-chip due to their size and because typical $\mathrm{Si}$ substrates are highly conductive. However, if the antenna can be realized on-chip, it can result in a fully integrated, low cost RF SoC with no bond wires and off-chip components. Antenna impedance can be utilized to replace the matching network interface between antenna and the RF circuits. Though the initial onchip antenna design has been reported almost a decade ago, not until recently, this concept has gained popularity in RF researchers. Many monolithic $\mathrm{Si}$ integrated antennas have been demonstrated in recent years [3-6]. The on-chip antenna design in literature is mostly restricted to higher frequencies like $10 \mathrm{GHz}$ and above due to large size of antennas at lower frequencies. On-chip antennas, as described in the literature, are either utilized to provide inter and intra- chip wireless interconnects [3] or for short-range air transmission [4]. The former antennas display high transmission loss and are not characterized for element gain and radiation pattern whereas the latter often utilize additional processing steps to increase the resistivity of Si substrates in an attempt to enhance the antenna performance. However, the major challenge is the poor radiation efficiency due to lossy $\mathrm{Si}$ substrate. In [5], an attempt has been made to improve the radiation efficiency and gain of on-chip antenna through an un-doped silicon substrate and a large silicon lens on the backside of the chip. Similarly, a high resistivity SiGe platform has been used in [6] to enhance the antenna gain. Recent proliferation of on-chip antennas has not been properly characterized, in particular, for their radiation pattern.

This paper is focused on the issues related to the on-chip antenna design, layout and characterization. A new test fixture is proposed to measure the gain and radiation pattern of the on-chip antennas in the anechoic chamber.

\section{Design AND LAyout CONSIDERATIONS}

The true advantage of on-chip antenna can be attained by its realization in standard CMOS process so that the RF circuits can be integrated with the back end digital blocks on the same chip. However, there are a number of challenges associated with this concept. 


\section{A. Incompatible Substrate}

In standard silicon process the substrate is lossy due to its low substrate resistivity, which often is less than $10 \Omega$-cm. Moreover, the high dielectric constant of silicon causes most of the power to be absorbed in the substrate. This is a major drawback for antenna implementation, as it does not allow the energy to radiate efficiently into free space.

\section{B. Implementation Limitations in CMOS}

Typically, in a custom process, on-chip antenna is implemented in the metallization layer on top of $\mathrm{SiO}_{2}$ that is above the $\mathrm{Si}$ substrate as shown in Fig. 1 (a). This ensures direct radiation in to free space. Such arrangement can also benefit from an off-chip ground to enhance radiation through reflection of the substrate-absorbed power. Though power lost in substrate and surface waves would still be a problem, on-chip antennas can be realized for shortrange communication [7]. Another option is to use the backside of the substrate for radiation [5]. Since most of the power is absorbed in the substrate anyways, it is wise to guide it from the backside of the substrate that does not contain an off-chip ground. Antennas may be employed at the interface of $\mathrm{Si}$ and $\mathrm{SiO}_{2}$ in this case. This approach is closer to a conventional CMOS process (shown in Fig. 1 (b)), where the stack-up restricts antenna implementation to metal layers within the oxide. A typical CMOS process has 6 to 8 metal layers embedded in the oxide which can be as thick as $15 \mu \mathrm{m}$. Top metal layers are generally thick $(\sim 4 \mu \mathrm{m})$ in order to fabricate on-chip inductors. Bottom metal layer, in general, is used as a ground shield for reducing losses of the on-chip inductors. However, the ground shield, if employed for on-chip antenna, would causes strong coupling and loss of energy because of proximity to antenna. Nonetheless, it has recently been shown that ground shield can improve antenna efficiency if a slot type antenna topology is chosen [8].

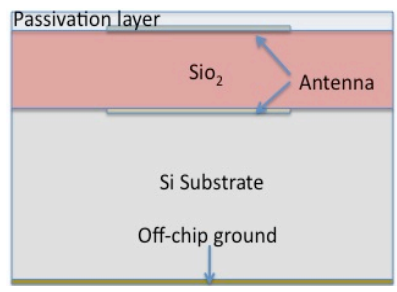

(a)

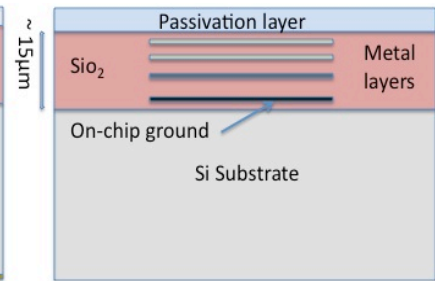

(b)
Figure 1. On-chip antenna realization in (a) custom setup for top and bottom-side radiation (b) standard CMOS

\section{Co-design with Circuits}

It is imperative for on-chip antenna design that it is suitable for integration with RF circuits on the same chip. This requires a co-design strategy for RF circuits and antenna. The antenna needs not to be matched to $50 \Omega$, infact, it is better to conjugate match it to the impedance of the RF circuit. In case, it is challenging to match the RF circuit impedance through mere changes in the antenna geometry, the RF circuits may also be tuned along with the antenna towards optimum impedance for both. This is the true advantage of a co-design strategy. At present, most of the modern RF circuits employ a differential architecture because of its better noise immunity. In that case, it is better to select a differential antenna topology, like dipole and loop, to avoid a balun in between the antenna and the circuits. Moreover, on-chip antennas perform better in terms of their radiation properties if placed close to the edges of the chip as compared to the center. This not only allows direct radiation to free space but also minimizes the interference from RF circuits.

\section{Design Rule Check Errors in Layout}

Since antennas are typically not on-chip, commercial CMOS processes do not have special metal layers or design rules for them. This makes it extremely difficult to resolve the Design Rule Check (DRC) errors because of the presence of on-chip antenna in the layout. Innovative solutions will have to be incorporated to resolve such errors until CMOS processes have advanced enough to accommodate efficient on-chip antennas. An example, in this regard, is the maximum permissible width of the CMOS metal layers. A typical process does not allow metal widths of more than $25 \mu \mathrm{m}$ to be connected to the transistors because of electrostatic discharge issues. However, this metal width is not sufficient for on-chip antenna design and affects the antenna gain in a negative way. If, now, the metal width is increased beyond this limit, it will result in the violation of DRC. A shortcut to resolve this issue is to provide a ground connection to the antenna in the top metal layer. This ground connection can be removed through microsurgery (laser) before the measurements. It was successfully implemented in one of our previous design [9], as shown in Fig. 2. 


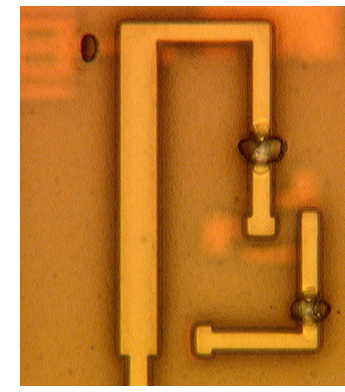

Figure 2. On-chip microsurgery (lasering)

\section{E. Coupling and Interference Issues}

Because of highly integrated designs, an important issue to consider is the parasitic coupling from circuits and nearby bond pads to the on-chip antenna and vice versa. This undesired coupling may deteriorate antenna's as well as circuits performance. It is important that circuit's placement with respect to antenna is carefully investigated in Electromagnetic (EM) simulations. Inductors placement is even more challenging as they also radiate somewhat. A smart technique to layout close by inductors is to ensure that currents in these inductors are oppositely directed. This will cancel the radiated fields and reduce the mutual coupling. Effects of nearby radiator element on circuits must be assessed in post layout circuit simulations. Unfortunately, no single simulation tool can handle such IC design as the on-chip antenna needs to be simulated in an EM simulator like HFSS ${ }^{\mathrm{TM}}$, whereas the circuit simulation and final layout has to be done in a circuit simulator like Cadence ${ }^{\mathrm{TM}}$. In addition to minimize coupling through air, undesired coupling through the substrate must also be blocked. This can be achieved through guard rings around circuits and inductors. Furthermore, to fulfill the fabrication foundry requirement of metal density in each layer, the final layout has to be filled with metal blocks. The challenge is that this metal fill cannot be planned until the layout is near completion as the final metal density is unknown at initial stages. These metal blocks, if placed close to on-chip antenna, can de-tune the antenna impedance. Therefore metal fill must be done with back and forth simulations in EM and circuit simulator.

In the nutshell, there still remain a number of challenges associated with on-chip antenna's realization in standard CMOS processes, both at design and layout levels. These may be resolved by incorporating adequate changes in modern CMOS processes.

\section{ON-ChIP ANTENNA CHARACTERIZATION}

\section{A. Radiation Pattern Characterization Challenges}

For a finite-dimensional substrate, the surfacewave can radiate from the edge of the chip result in an erroneous radiation pattern. Similar problem can occur due to interference of circuits and antennas on a single chip. This makes it extremely important to characterize the radiation pattern of on-chip antenna to assess its true performance. Unfortunately, these measurements are not simple and require custom setup. It is for this reason that most of the on-chip antenna work done recently, does not characterize them for their radiation properties $[8,10]$.

On-chip antenna radiation characteristics cannot be measured in an anechoic chamber unless the chip is mounted on a board or a special test fixture. Mounting the chip on a board, as shown in Fig. 3 (a), results in undesirable radiation pattern because of blocking and interference from other components [9]. An alternate solution is to measure the radiation pattern on the bare die, as shown in Fig. 3 (b). This requires a custom setup with a moveable microscope and careful use of probes. However, it is challenging to measure the radiation pattern in all planes accurately with this technique. Another issue is that the metallic chuck of the probe station acts as a ground for the antennas and results in flawed radiation characterization. Ideally, the wafer or chip should either be exposed to free space or placed on a $\mathrm{RF}$ insulator, while being characterized for its radiation properties.

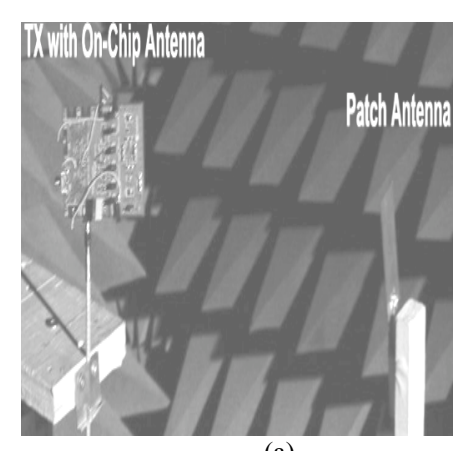

(a)

Figure 3. On-chip atenna radiation pattern measurements on (a) Printed Circuit Board (b) on-wafer

\section{B. On-wafer Radiation Characterization Fixture}

For accurately on-wafer testing of the on-chip antennas with the conventional setup in an anechoic chamber, a novel test fixture is proposed as shown in Fig. 4. The design is generic enough to permit 
measurements on both sides of differently sized wafers. It is fabricated with a low dielectric constant material duroid $\left(\varepsilon_{\mathrm{r}} \sim 2\right)$ to ensure minimum reflections and interference. The test fixture is composed of three disc shaped (near circular) duroid based Dielectric Plates (DP) with custom holes in them to accommodate wafer in a fashion that it is exposed to free space from both sides.

Both DP1 and DP2 have dielectric removed from the center to form a hole with diameter increasing from the bottom to the top. This creates a cavity each in DP1 and DP2, with a hole in the center. The edges of these cavities can support another DP or the wafer. DP1 being the thickest is also used to mount the RF probe and the position controller. DP2 is placed on the edges of DP1 cavity in a way that it is almost suspended in free space. The wafer is placed on the edges of DP2 cavity in a similar fashion and most of it is suspended in air. DP3 is placed on top of the wafer and it also has a hole in the center but instead of a cavity it has extended fingers to hold the wafer in place from three sides. The fourth side is left open for RF probe access. The wafer is sandwiched between DP2 and DP3, which can be fabricated with different diameter holes to accommodate different sizes of wafers. DP3 is fixed to DP2 through duroid-based screws, whereas DP2 is screwed to DP1. The RF probe can access till the center of a six-inch wafer through its position controller. In order to characterize test structures on the other half, the wafer can be rotated towards the RF probe. The complete fixture rests on four duroid based dielectric post that are fixed to a metallic holder plate, which in turn is mounted on the characterization tower in the anechoic chamber.

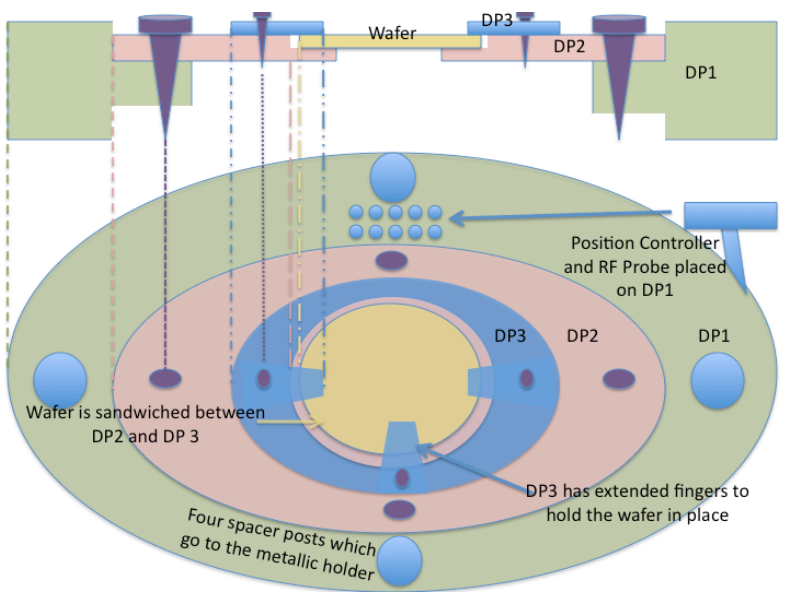

(a)

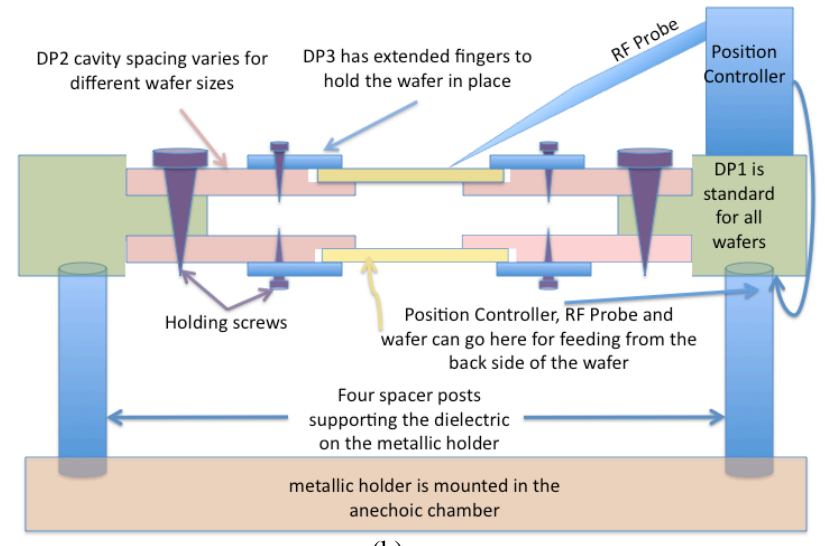

(b)

Figure 4. On-wafer test ficture (a) crossection and top view (b) complete crosssection view with backside feed arrangement and posts

These posts ensure that there is enough separation (in terms of electrical length) between the antenna and the metallic holder plate. Again these posts can be of different heights to allow characterization at different frequencies. There is a provision to test the antennas, which are fed from the backside of the wafer (lower wafer in Fig. 4 (b)). In that case, the RF probe and the position controller are placed in an inverted fashion. Obviously, the top wafer and DPs are not present in this case.

The photograph of the fabricated test fixture will be included in the final manuscript.

\section{REFERENCES}

[1] A. A. Abidi, "RF CMOS comes of age," Solid-State Circuits, IEEE Journal of, vol. 39, pp. 549-561, 2004.

[2] L. Wang, et.al., "A sensor system on chip for wireless microsystems," Circuits and Systems, 2006. ISCAS 2006. Proceedings. 2006 IEEE International Symposium on, pp. 4 pp.-858, 2006.

[3] Kihong Kim, Hyun Yoon and K. K. O, "On-chip wireless interconnection with integrated antennas," Electron Devices Meeting, 2000. IEDM Technical Digest. International, pp. 485-488, 2000.

[4] K. T. Chan, et.al., "Integrated antennas on $\mathrm{Si}$ with over $100 \mathrm{GHz}$ performance, fabricated using an optimized proton implantation process," Microwave and Wireless Components Letters, IEEE, vol. 13, pp. 487-489, 2003.

[5] A. Babakhani, et.al., "A 77-GHz Phased-Array Transceiver With OnChip Antennas in Silicon: Receiver and Antennas," Solid-State Circuits, IEEE Journal of, vol. 41, pp. 2795-2806, 2006.

[6] E. Ojefors, et.al., "Monolithic Integration of a Folded Dipole Antenna With a 24-GHz Receiver in SiGe HBT Technology," Microwave Theory and Techniques, IEEE Transactions on, vol. 55, pp. 1467-1475, 2007.

[7] A. Shamim, L. Roy, N. Fong and N. G. Tarr, "24 GHz On-Chip Antennas and Balun on Bulk Si for Air Transmission," Antennas and Propagation, IEEE Transactions on, vol. 56, pp. 303-311, 2008.

[8] Dan Shi, N. Behdad, Jia-Yi Chen and M. P. Flynn, "A 5GHz fully integrated super-regenerative receiver with on-chip antenna in $0.13 \mu \mathrm{m}$ CMOS," VLSI Circuits, 2008 IEEE Symposium on, pp. 34-35, 2008.

[9] A. Shamim, et.al., "A CMOS active antenna/inductor for System on a Chip (SoC) applications," Antennas and Propagation Society International Symposium, IEEE , pp.1-4, July 2008

[10] Wen-Yan Yin; et.al, "Performance of Intra-Chip Wireless Interconnect Using On-Chip Antennas and UWB Radios," Antennas and Propagation, IEEE Transactions on, vol.57, no.9, pp.2756-2762, Sept. 2009. 